21st Particles and Nuclei International Conference (PANIC 2017)

International Journal of Modern Physics: Conference Series

Vol. 46 (2018) 1860037 (6 pages)

(C) The Author(s)

DOI: $10.1142 / \mathrm{S} 2010194518600376$

\title{
Study of Heavy Strange Baryons in a Hypercentral Quark Model
}

\author{
Zahra Ghalenovi \\ Department of Physics, Kosar University of Bojnord, Bojnord, Iran
}

Published 3 May 2018

\begin{abstract}
In this work, we study the properties of the heavy baryons with strangeness employing a constituent quark model in the hypercentral approach. The potential model considers the interactions arising the one-gluon exchange, Goldstone boson exchange and confinement, aspects of underlying theory, quantum chromodynamics (QCD). By solving three-body Schrodinger equation of baryonic system, we obtain the ground as well as the corresponding energy eigenvalues of the system. Using the obtained energies, we calculate the baryon spectrum. We extend our scheme to predict the radiative decay width of the charm baryons. A comparison of our results with those of other works and experimental data is also presented.
\end{abstract}

Keywords: Potential model; Hypercentral approach; Non-relativistic limit; Baryon properties.

\section{Introduction}

During last few years a significant experimental progress and theoretical activities have been achieved in charmed and bottom baryon spectroscopy [1-3]. The masses of most of heavy baryons have not been measured yet experimentally. Thus the recent predictions about the heavy baryon masses have become a subject of renewed interest. The study of heavy flavour baryons provide excellent laboratory to understand the dynamics of light quarks in the vicinity of heavy flavour quarks in bound states.

In this work we use the non-relativistic quark model to calculate the ground state masses of the heavy baryons. In section 2 we solve the non-relativistic three-body problem in the hypercentral approach and find the eigenfunctions and the eigenvalues of Schrödinger equation for the baryons. In section 3 the exchange interactions, boson and one-gluon exchange interactions, are studied. We calculate the masses of the heavy strange baryons and present our results in section 4 . In section 5 we predict the radiative decay width for the $\Xi_{c}^{*+} \rightarrow \Xi_{c}^{+}$and $\Xi_{c}^{* 0} \rightarrow \Xi_{c}^{0}$ processes.

This is an Open Access article published by World Scientific Publishing Company. It is distributed under the terms of the Creative Commons Attribution 4.0 (CC-BY) License. Further distribution of this work is permitted, provided the original work is properly cited. 


\section{Z. Ghalenovi}

\section{The Hypercentral Scheme for the Baryonic Systems}

In the hypercentral constituent quark model (hCQM), the quark potential, $V$ is assumed to depend only on the hyperradius $x$ only that is to be hypercentral and therefore the remaining hyperradial part of the wave function is determined by hypercentral Schrödinger equation as follows $[4-6]$

$$
\left[\frac{d^{2}}{d x^{2}}+\frac{5}{x} \frac{d}{d x}-\frac{\gamma(\gamma+4)}{x^{2}}\right] \psi_{\gamma}(x)=-2 m\left[E_{\gamma}-V(x)\right] \psi_{\gamma}(x)
$$

where $x$ is the hyperradius and $\gamma$ is the grand angular quantum number. $\psi_{\gamma}(x)$ and $E_{\gamma}$ are the hyperradial part of the wave function and the energy eigenvalues and $m$ is the reduced mass.

In this model the three-quark potentials $V(x)$ is considered as

$$
V(x)=a x^{2}+V_{0}
$$

where $a$ and $V_{0}$ are arbitrary parameters. The transformation

$$
\psi(x)=x^{-5 / 2} \phi(x)
$$

reduces the Schrödinger equation (1) to the form

$$
\frac{d^{2}}{d x^{2}} \varphi_{\gamma}(x)+\left[\varepsilon_{\gamma}-2 \max ^{2}-\frac{(2 \gamma+3)(2 \gamma+5)}{4 x^{2}}\right] \varphi_{\gamma}(x)=0 .
$$

where

$$
\varepsilon_{\gamma}=2 m\left(E_{\gamma}-V_{0}\right) .
$$

The effective potential $U_{\gamma}(x)$ reads [7]

$$
U_{\gamma}(x)=-2 \max ^{2}-\frac{(2 \gamma+3)(2 \gamma+5)}{4 x^{2}}
$$

In order to solve Eq. (4), we perform a Taylor expansion of $U_{\gamma}(x)$ around $x=x_{\gamma}$,

$$
U_{\gamma}(x) \approx U_{\gamma}\left(x_{\gamma}\right)+\Omega_{\gamma}^{2}\left(x-x_{\gamma}\right)^{2},
$$

where $x_{\gamma}$ is such that $d U_{\gamma}(x) /\left.d x\right|_{x=x_{\gamma}}=0$ and

$$
\Omega_{\gamma}^{2}=\left.\frac{1}{2 !} \frac{d^{2} U_{\gamma}(x)}{d x^{2}}\right|_{x=x_{\gamma}} .
$$

Substituting Eq. (7) for $U_{\gamma}$, Eq. (6), into Eq. (4) we find

$$
\frac{d^{2}}{d x^{2}} \varphi_{\gamma}(x)-\Omega_{\gamma}^{2}\left(x-x_{\gamma}\right)^{2} \varphi_{\gamma}(x)=-\left[\varepsilon_{\gamma}-U_{\gamma}\left(x_{\gamma}\right)\right] \varphi_{\gamma}(x)
$$

which is the well-known equation for a one-dimensional harmonic oscillator. Namely, for a particle with mass $m$, oscillation frequency $\omega^{\prime}$, energy eigenvalues $\varepsilon^{\prime}=$ $\left(n+\frac{1}{2}\right) \hbar \omega^{\prime}$, and spatial wave function $\phi(x)$, the one-dimensional harmonic oscillator equation reads:

$$
\frac{d^{2}}{d x^{2}} \phi(x)-\frac{m^{2} \omega^{\prime 2} x^{2}}{\hbar^{2}} \phi(x)=-\frac{2 m \varepsilon^{\prime}}{\hbar^{2}} \phi(x) .
$$


We consider here the ground state baryons $(\gamma=n=0)$. In this way, upon a comparison of Eq. (9) with Eq. (10), we have:

$$
\Omega_{0}=\frac{m \omega^{\prime}}{\hbar}, \quad \varepsilon_{0}-U_{0}\left(x_{0}\right)=\frac{2 m \varepsilon^{\prime}}{\hbar^{2}} .
$$

Finally, the ground-state energy eigenvalue $E_{0}$ is obtained using Eq. (5):

$$
E_{0}=V_{0}+\frac{1}{2 m}\left[U_{0}\left(x_{0}\right)+\Omega_{0}\right]
$$

with the corresponding ground-state wave function

$$
\varphi_{0}(x)=\sqrt{\frac{\Omega_{0}}{\pi}} e^{-\frac{1}{2} \Omega_{0} x^{2}},
$$

where the constant term in front is a normalization constant.

\section{Chiral Dynamics and the Quark Model}

Chiral symmetry suggests to divide the quarks into two different sectors: light quarks $(\mathrm{u}, \mathrm{d}$ and $\mathrm{s}$ ) where chiral symmetry is spontaneously broken, and heavy quarks (c and b) where the symmetry is explicitly broken. Therefore for the light quark sector baryons can be described as systems of confined constituent quarks interacting through gluon and boson exchanges, and for the heavy quark sector described as systems of confined current quarks interacting through gluon exchanges [8]

$$
V_{q_{i} q_{j}}=\left\{\begin{array}{l}
\left(q_{i} q_{j}\right)=(n n, n s, s s) \Rightarrow V_{C O N}+V_{O G E}+V_{\chi}, \\
\left(q_{i} q_{j}\right)=(n Q, s Q, Q Q) \Rightarrow V_{C O N}+V_{O G E}
\end{array}\right.
$$

Where $n$, s and Q refer to the light quarks ( $u$ and d), strange quark and heavy quarks (c and b) respectively. $V_{\chi}$ stands for the chiral part of the interaction we will discuss below. For energies below the scale of spontaneous chiral symmetry breaking, baryons can be viewed as systems of three quarks interacting via exchange of Goldstone bosons (pseudoscalar mesons). In the $S U(3)_{F}$ invariant approximation the pseudoscalar exchange interaction splits the multiplets of $S U(6)_{S F} \times U(6)_{\text {conf }}$ in the spectrum to multiplets of $S U(3)_{F} \times S U(2)_{s} \times U(6)_{c o n f}$. The position of these multiplets differ in the baryon sectors with different strangeness because of the mass splitting of the pseudoscalar octet and the different constituent masses of the $\mathrm{u}, \mathrm{d}$ and s quarks that breaks $S U(3)_{F}$ flavor symmetry. The simple representation of the quark-quark meson exchange interactions in the $S U(3)_{F}$ invariant limit is

$$
V_{\chi} \approx-\sum_{i<j} V\left(\vec{r}_{i j}\right) \vec{\lambda}_{i}^{F} \cdot \vec{\lambda}_{j}^{F} \vec{\sigma}_{i} \cdot \vec{\sigma}_{j}
$$

Where the $\lambda, s$ are $\mathrm{SU}(3)$ flavour Gell-Mann matrices and the $i$ and $j$ sums run over the constituent quarks [9]. The radial part $V(r)$ behaves as the usual Yukawa behavior at long range, but at short range behaves as a smeared version of the $\delta$ function term in the Yukawa interaction for pseudoscalar exchange. 


\section{Z. Ghalenovi}

The form of OGE potential for the ground state baryons is follows

$$
V^{O G E}\left(\vec{r}_{i j}\right)=\frac{\alpha_{s}}{4} \lambda_{i}^{c} \cdot \lambda_{j}^{c} \times\left[\frac{1}{r_{i j}}-\frac{1}{6 m_{i} m_{j}}\left(\vec{\sigma}_{i} \cdot \vec{\sigma}_{j}\right) \times \delta\left(r_{i j}\right)\right]
$$

Where $\lambda^{c}$ are the $S U(3)$ color matrices, $\alpha_{s}$ is the quark-gluon coupling constant and $m_{i}$ is the $i$ th quark mass. The $\delta$ function is regularized in a suitable way. The first term (18) is the columbic interaction, and the second term is the most important component and called color-magnetic interaction between two quarks. The strong coupling constant for each flavor sector is different [1]. In this model $\alpha_{s}$ depends on the quark masses:

$$
\alpha_{s}(\mu)=\frac{\alpha_{0}}{\ln \left[\left(\mu^{2}+\mu_{0}^{2}\right) / \gamma_{0}^{2}\right]}
$$

where $\mu$ is the reduced mass of the interacting quarks and $\alpha_{0}=2.118, \mu_{0}=$ $36.976 \mathrm{MeV}$, and $\gamma_{0}=0.113 \mathrm{fm}^{-1}$.

The main idea of this model is to study the heavy baryon systems based on the exchange interactions in the hypercentral approach. The pseudoscalar meson exchange interaction has two distinct radial parts: a long-range Yukawa potential tail and a short-range part, $\delta$ function. It is the latter which plays a major role in describing the baryon spectra in the frame of Goldstone boson exchange models [10]. The delta function appearing in (15) and (18) needs to be regularized for practical calculations. In this model we employ the Gussian-type smearing of the delta function for the exchange interactions (GBE and OGE) as [11]

$$
\delta(r) \approx \frac{\xi_{i}}{\left(\sqrt{\pi} r_{i}\right)^{3}} \exp \left(-\frac{r^{2}}{r_{i}^{2}}\right),
$$

where $r_{i}$ and $\xi_{i}$ are constant and has different values for the GBE and OGE interactions. Therefore, the radial part of the GBE in (15) is assumed to be as following

$$
V(r) \approx \frac{\xi_{G B E}}{\left(\sqrt{\pi} r_{G B E}\right)^{3}} \exp \left(-\frac{r^{2}}{r_{G B E}^{2}}\right) .
$$

\section{Heavy Baryons Masses}

Baryon mass is obtained by sum of the quarks mass and energy of perturbed system:

$$
M_{\text {baryon }}=\sum_{i=1}^{3} m_{i}+E_{0 \gamma}+\delta M_{\chi}
$$

Where the boson and gluon exchange interactions are treated in the first order perturbation theory:

$$
\delta M_{\chi}=\left\langle\psi\left|H_{\chi}\right| \psi\right\rangle
$$

We consider the interaction potentials in $S U(3)_{F}$ invariant limit. Then the constituent quark masses (for the light quark sector) would be equal, $m_{u}=m_{d}=m_{s}$. The potential parameters and quark masses are taken from Refs. $[4,12,13]$ respectively. We use the experimental masses of $\Sigma(1193)$ and $\Xi(1318)$, to determine $\xi_{i}$ 
Table 1. Quark-model parameters.

\begin{tabular}{l|l}
\hline$m_{u}=m_{d}=m_{s}$ & $313 \mathrm{MeV}$ \\
$m_{c}$ & $1600 \mathrm{MeV}$ \\
$m_{b}$ & $4980 \mathrm{MeV}$ \\
$a$ & $2.71 \mathrm{fm}^{-2}$ \\
$r_{G B E}$ & $3.64 \mathrm{fm}^{-1}$ \\
$\xi_{G B E}$ & $0.172 \mathrm{fm}^{-1}$ \\
$r_{O G E}$ & $0.518 \mathrm{fm}^{-1}$ \\
$\xi_{O G E}$ & $16.89 \mathrm{fm}^{-1}$ \\
\hline
\end{tabular}

Table 2. Heavy strange baryon masses (in $\mathrm{MeV}$ ).

\begin{tabular}{l|l|l|l|l|l}
\hline Baryon & Present work & $\operatorname{Exp}[17-19]$ & {$[3]$} & {$[21]$} & {$[20]$} \\
\hline$\Xi_{c}^{0}$ & 2478 & 2476 & 2494 & 2529 & - \\
$\Xi_{c}^{* 0}$ & 2632 & 2646 & 2680 & 2604 & - \\
$\Omega_{c}^{0}$ & 2680 & 2699 & 2696 & 2601 & 2718 \\
$\Omega_{c}^{* 0}$ & 2775 & 2768 & 2757 & 2684 & 2776 \\
$\Xi_{b}^{-}$ & 5833 & 5792 & 5887 & - & 5806 \\
$\Xi_{b}^{*-}$ & 5987 & - & 5943 & - & 5980 \\
$\Omega_{b}^{-}$ & 6084 & 6054 & 6005 & - & 6081 \\
$\Omega_{b}^{*-}$ & 6129 & - & 6065 & - & 6102 \\
\hline
\end{tabular}

(see Table 1). The obtained baryon masses in our model are listed in Table 2 and compared with the experimental data $[17-19]$ or those of other works $[3,20,21]$.

\section{Radiative decay width}

The radiative decay width of the baryons is given by [14-16]

$$
\Gamma_{R}=\frac{q^{3}}{4 \pi} \frac{2}{2 J+1} \frac{e^{2}}{m_{p}^{2}}\left|\mu_{\frac{3}{2}}{ }^{+} \rightarrow \frac{1}{2}+\right|^{2},
$$

where $m_{p}$ is the proton mass, $\mu_{\frac{3}{2}}{ }^{+} \frac{1}{2}^{+}$is the radiative transition magnetic moments and $q$ is the photon energy.

We calculate the transition magnetic moments by sandwiching $\Sigma_{i} \mu_{i} \sigma_{i}$ between the appropriate $3 / 2^{+}$and $1 / 2^{+}$baryon wave functions. The transition magnetic moments for $\frac{3}{2}^{+} \rightarrow \frac{1}{2}^{+}$are computed as

$$
\mu_{\frac{3}{2}}^{+} \rightarrow \frac{1}{2}^{+}=\Sigma_{i}<\phi_{s f}^{2^{3}}\left|\mu_{i} \sigma_{i}\right|_{s f}^{\frac{1}{2}^{+}}>\text {. }
$$

$<\phi_{s f}^{\frac{3}{2}} \mid$ represents the spin-flavour wave function of the quark composition for

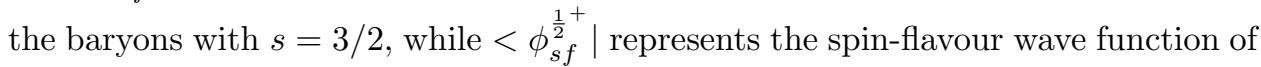
the quark composition for the baryons with $s=1 / 2$ (for details, see Refs $[14,15]$ ). Using Eq. (22) we obtain the radiative decay widths for the $\Xi_{c}^{*+} \rightarrow \Xi_{c}^{+}$and $\Xi_{c}^{* 0} \rightarrow \Xi_{c}^{0}$ processes as follows

$$
\Gamma_{\Xi_{c}^{*+} \rightarrow \Xi_{c}^{+}}=68.1 \mathrm{KeV}, \quad \Gamma_{\Xi_{c}^{* 0} \rightarrow \Xi_{c}^{0}}=0.50 \mathrm{KeV} .
$$




\section{Z. Ghalenovi}

Our results are in agreement with the ones of Refs. [14,22] who obtained 63.8 $\mathrm{KeV}$ and $75.6 \mathrm{KeV}$ for $\Gamma_{\Xi_{c}^{*+} \rightarrow \Xi_{c}^{+}}$and $0.30 \mathrm{KeV}$ and $0.90 \mathrm{KeV}$ for $\Gamma_{\Xi_{c}^{* 0} \rightarrow \Xi_{c}^{0}}$ respectively.

\section{Conclusion and Discussion}

The heavy baryons are studied as systems of three constituent quarks in the hypercentral approach. This model takes into account the one-gluon exchange, boson exchange and confinement. By solving the Schrödinger equation for the confining potential, we are able to obtain the eigenfunctions and eigenvalues of the baryons. By considering the exchange interactions as perturbation, we can calculate the heavy baryon masses. we also predict the radiative decay widths for the charm strange baryons. A comparison between our results and other results shows that our model can describe the baryon properties well. This model can be extended to study meson spectra and baryon-baryon interactions with chiral dynamics.

\section{References}

1. A. Valcarce, H. Garcilazo and J. Vijande, Phys. Rev. C 72, 025206 (2005).

2. Z. Ghalenovi, A. A. Rajabi and A. Tavakolinezhad, Int. J. Mod. Phys. E 21, (2012) 1250057 .

3. B. Patel, A. Kumar Rai, J. Phys. G: Nucl. Part. Phys. 35, 065001 (2008).

4. Z. Ghalenovi and M. Moazzen, Eur. Phys. J. Plus 132, no. 8, 354 (2017).

5. Z. Ghalenovi, A. A. Rajabi, S. x. Qin and D. H. Rischke, Mod. Phys. Lett. A 29 (2014) 1450106.

6. Z. Ghalenovi, A. A. Rajabi and A. Tavakolinezhad, Int. J. Mod. Phys. E 21 (2012) 1250057 .

7. H. Hassanabadi et al., Few-Body Syst. 48, (2010) 53.

8. J. Vijande and A. Valcarce, Phys. Lett. B 677, 36 (2009).

9. L. Ya. Glozman, D. O. Riska,Phys. Rep. C 268, 263 (1996).

10. P. Stassart, Fl. Stancu and J.-M. Richard, J. Phys. G 26, 397 (2000).

11. L.Ya. Glozman et al., Phys. Rev. C 57, 3406 (1998).

12. Bhavyashri, K. B. Vijaya Kumar, Arxiv: hep-ph/0811.4308v2 (2008).

13. Z. Ghalenovi and A. Akbar Rajabi, Eur. Phys. J. Plus 127, 141 (2012).

14. A. Majethiya, B. Patel and P. C. Vinodkumar, Eur. Phys. J. A 42 (2009) 213.

15. Z. Ghalenovi and M. Moazzen, Int. J. Mod. Phys. E 26, 07 (2017).

16. Z. Ghalenovi, arXiv:1707.01319 [hep-ph].

17. W. M. Yao et al. (Particle Data Group), J. Phys. G 33, 1 (2006).

18. T. Aaltonen et al.(CDF collaboration), Phys. Rev. Lett 99, 202001 (2007).

19. T. Aaltonen et al, Phys. Rev. D 80, 072003 (2009).

20. W. Roberts, Muslema pervin, arXiv:0711.2492v2 [nucl-th] (2008).

21. B. Patel, A. Majethiya and P.C. Vinodkumar, Phys. At. Nucl. 65, 917 (2002).

22. 14. Fayyazuddin and Riazuddin, Mod. Phys. Lett. A 12, 1791 (1997). 